Journal of Mathematics and Informatics

Vol. 10, 2017, 49-55

ISSN: 2349-0632 (P), 2349-0640 (online)

Published 11 December 2017

www.researchmathsci.org

DOI: http://dx.doi.org/10.22457/jmi.v10a7

Journal of

Mathematics and

Informatics

\title{
On the Non-Homogeneous Ternary Quadratic Equation
}

$$
2\left(x^{2}+y^{2}\right)-3 x y+(x+y)+1=z^{2}
$$

\section{A. Priya ${ }^{1}$ and $S$. Vidhyalakshmi ${ }^{2}$}

${ }^{1}$ Department of Mathematics, Shrimati Indira Gandhi College, Trichy-2, Tamilnadu

INDIA, e.mail: priyaalagesan96@gmail.com

${ }^{2}$ Department of Mathematics, Shrimati Indira Gandhi College, Trichy-2, Tamilnadu

INDIA, e.mail: vidhyasigc@gmail.com

Received 15 November 2017; accepted 04 December 2017

Abstract. The non-homogeneous quadratic Diophantine equation represented by $2\left(x^{2}+y^{2}\right)-3 x y+(x+y)+1=z^{2}$ is studied for its non-zero distinct integer solutions. Four different sets of distinct integer solutions to the above equation are obtained. A few interesting relations between the solutions and special polygonal numbers are presented.

Keywords: Non-homogeneous quadratic, ternary quadratic, integer solutions.

\section{AMS Mathematics Subject Classification (2010): 11D09}

\section{Introduction}

The Diophantine equation offer an unlimited field for due to their variety $[1-3]$. In particular, one may refer [4-14] for quadratic equations with three unknowns. This communication concerns with yet another interesting homogeneous quadratic equation with three unknowns given by $2\left(x^{2}+y^{2}\right)-3 x y+(x+y)+1=z^{2}$ for determining its infinitely many non-zero integral points. Also, a few interesting relations among the solutions are presented.

\section{Notations}

$$
\begin{aligned}
& t_{m, n}=\mathrm{n}^{\text {th }} \text { term of a regular polygon with m sides } \\
= & n\left(1+\frac{(n-1)(m-2)}{2}\right)
\end{aligned}
$$

\section{Method of analysis}

The ternary quadratic Diophantine equation to be solved for its non-zero distinct integral solution is

$$
2\left(x^{2}+y^{2}\right)-3 x y+(x+y)+1=z^{2}
$$




\section{A.Priya and S.Vidhyalakshmi}

Introduction of the linear transformations $(u \neq v \neq 0)$

$x=u+v, y=u-v$

in (1) leads to

$$
U^{2}+7 v^{2}=z^{2}
$$

where $U=u+1$

Different patterns of solutions of (1) are presented below.

\subsection{PATTERN-1}

Assume

$$
z=a^{2}+7 b^{2}
$$

where $\mathrm{a}$ and $\mathrm{b}$ are non-zero distinct integers.

Write 1 as

$$
1=\frac{(3+i \sqrt{7})(3-i \sqrt{7})}{16}
$$

Using (4) and (5) in (3) and employing the method of factorization (3) is written as

$$
(U+i \sqrt{7} v)(U-i \sqrt{7} v)=\frac{(3+i \sqrt{7})(3-i \sqrt{7})}{16}(a+i \sqrt{7} b)^{2}(a-i \sqrt{7} b)^{2}
$$

Equating the positive and negative factors, the resulting equations are

$$
\begin{aligned}
& U+i \sqrt{7} v=\frac{(3+i \sqrt{7})}{4}(a+i \sqrt{7} b)^{2} \\
& U-i \sqrt{7} v=\frac{(3-i \sqrt{7})}{4}(a-i \sqrt{7} b)^{2}
\end{aligned}
$$

Equating the real and imaginary parts in (6),

$$
\begin{aligned}
& U=\frac{1}{4}\left(3 a^{2}-21 b^{2}-14 a b\right) \\
& v=\frac{1}{4}\left(a^{2}-7 b^{2}+6 a b\right)
\end{aligned}
$$

Replacing $a, b$ by $2 A, 2 B$ respectively, we get

$$
U=3 A^{2}-12 B^{2}-14 A B
$$

$v=A^{2}-7 B^{2}+6 A B$

In view of (3a) 
On the Non-Homogeneous Ternary Quadratic Equation $2\left(x^{2}+y^{2}\right)-3 x y+(x+y)+1=z^{2}$ $u=3 A^{2}-21 B^{2}-14 A B-1$

Substituting the values of $u$ and $v$ in (2), we get

$$
\begin{aligned}
& x=x(A, B)=4 A^{2}-28 B^{2}-8 A B-1 \\
& y=y(A, B)=2 A^{2}-14 B^{2}-20 A B-1
\end{aligned}
$$

Thus (8),(9) and(4) represents non-zero distinct integral solutions of (1) in two parameters.

\section{Properties observed are as follows}

1. $6\left(t_{18, \alpha^{2}}-x\left(\alpha^{2}, 1\right)-z\left(\alpha^{2}, 1\right)-1\right)$ is a nasty number.

2. $\left.2 y(A, 1)+z(A, 1)-t_{18, A}+2 \equiv 0(\bmod 3)\right)$

3. $(9-3 x(A, A+1))$ is a nasty number

4. $x(A, 1)-y(A, 1)+z(A, 1)-t_{18, A}-14 \equiv 0(\bmod 3)$

5. $x(A, A+1)+y(A, A+1)+z(A, A+1)+t_{66, A}+16 \equiv 2(\bmod 5)$

\subsection{PATTERN-2}

Write 1 as

$$
1=\frac{(1+i 3 \sqrt{7})(1-i 3 \sqrt{7})}{64}
$$

Using (4) and (10) in (3) and employing the method of factorization(3) is written as

$$
(U+i \sqrt{7} v)(U-i \sqrt{7} v)=\frac{(1+i 3 \sqrt{7})(1-i 3 \sqrt{7})}{64}(a+i \sqrt{7} b)^{2}(a-i \sqrt{7} b)^{2}
$$

Equating the positive and negative factors, the resulting equations are

$$
\begin{aligned}
& U+i \sqrt{7} v=\frac{(1+i 3 \sqrt{7})}{8}(a+i \sqrt{7} b)^{2} \\
& U-i \sqrt{7} v=\frac{(1-i 3 \sqrt{7})}{8}(a-i \sqrt{7} b)^{2}
\end{aligned}
$$

Equating the real and imaginary parts in (11),

$$
U=\frac{1}{8}\left(a^{2}-7 b^{2}-42 a b\right)
$$


A.Priya and S.Vidhyalakshmi

$v=\frac{1}{8}\left(3 a^{2}-21 b^{2}+2 a b\right)$

Replacing $a, b$ by $4 A, 4 B$ respectively, we get

$U=2 A^{2}-14 B^{2}-84 A B$

$v=6 A^{2}-42 B^{2}+4 A B$

In view of (3a)

$u=2 A^{2}-14 B^{2}-84 A B-1$

Substituting the values of $u$ and $v$ in (2), we get

$$
\begin{aligned}
& x=x(A, B)=8 A^{2}-56 B^{2}-80 A B-1 \\
& y=y(A, B)=-4 A^{2}+28 B^{2}-88 A B-1
\end{aligned}
$$

Thus (13),(14) and(4) represents non-zero distinct integral solutions of(1) in two parameters.

\section{Properties observed are as follows}

1. $x(A, 1)-y(A, 1)-t_{10, A}+84 \equiv 1(\bmod 3)$

2. $\left.y(2 B-1, B)+z(2 B-1, B)-t_{26, B}-11 \equiv 0(\bmod 3)\right)$

3. $x(A, 1)-y(A, 1)+z(A, 1)-t_{50, A}-28 \equiv 0(\bmod 3)$

4. $x(A, 1)-y(A, 1)+z(A, 1)-t_{58, A}-28 \equiv 0(\bmod 5)$

5. $y(A, 1)+t_{10, A}+27 \equiv 1(\bmod 3)$

\subsection{PATTERN-3}

(3) is written in the form of ratio as

$$
\frac{z+U}{7 v}=\frac{v}{z-U}=\frac{m}{n}, n \neq 0
$$

which is equivalent to the system of equations,

$$
\begin{aligned}
& n z+n U-7 m v=0 \\
& m z-m U-n v=0
\end{aligned}
$$

Applying the method of cross multiplication for solving (16) and (17), we have

$$
\begin{aligned}
& U=7 m^{2}-n^{2} \\
& v=2 m n
\end{aligned}
$$


On the Non-Homogeneous Ternary Quadratic Equation $2\left(x^{2}+y^{2}\right)-3 x y+(x+y)+1=z^{2}$

$z=7 m^{2}+n^{2}$

In view of (3a) we get

$u=7 m^{2}-n^{2}-1$

Substituting the values of $u$ and $v$ in (2)

$$
\begin{aligned}
& x=x(m, n)=7 m^{2}-n^{2}+2 m n-1 \\
& y=y(m, n)=7 m^{2}-n^{2}-2 m n-1
\end{aligned}
$$

Thus (18) and (19) represent the integer solutions to (1)

\section{Properties observed are as follows}

1. $x(m, m+1)-y(m, m+1)=8 t_{3, m}$

2. $y(m, 1)+z(m, 1)-t_{30, m}+1 \equiv 0(\bmod 11)$

3. $x(2 n-1, n)-y(2 n-1, n)=4 t_{6, n}$

4. $y(m, 1)-t_{16, n}+2 \equiv 0(\bmod 2)$

5. $x(m, m+1)+z(m, m+1)-t_{34, m}+1 \equiv 2(\bmod 3)$

\subsection{PATTERN-4}

(3) is written in the form of ratio as

$$
\frac{z+U}{v}=\frac{7 v}{z-U}=\frac{m}{n}, n \neq 0
$$

which is equivalent to the system of equations

$$
\begin{aligned}
& n z+n U-m v=0 \\
& m z-m U-7 n v=0
\end{aligned}
$$

Applying the method of cross multiplication for solving (21) and (22), we have

$$
\begin{aligned}
& U=m^{2}-7 n^{2} \\
& v=2 m n \\
& z=m^{2}+7 n^{2}
\end{aligned}
$$

In view of (3a) we get,

$u=m^{2}-7 n^{2}-1$

Substituting the values of $u$ and $v$ in (2) 
A.Priya and S.Vidhyalakshmi

$$
\begin{aligned}
& x=x(m, n)=m^{2}-7 n^{2}+2 m n-1 \\
& y=y(m, n)=m^{2}-7 n^{2}-2 m n-1
\end{aligned}
$$

Thus (23) and (24) represent the integer solutions to (1)

\section{Properties observed are as follows}

1. $6\left(t_{6, \alpha^{2}}-y\left(\alpha^{2}, 1\right)-z\left(\alpha^{2}, 1\right)-1\right)$ is a nasty number.

2. $6(6 y(m, 1)+3 z(m, 1)+31)$ is a nasty number

3. $x(m, m+1)+t_{10, m}+8 \equiv 0(\bmod 5)$

4. $x(m, 1)+y(m, 1)+z(m, 1)+9=3 t_{4, m}$

5. $x(m, 1)+z(m, 1)-t_{6, m}+1 \equiv 0(\bmod 3)$

\section{Conclusion}

In this paper, we have obtained infinitely many non-zero distinct integer solutions to the ternary quadratic Diophantine equation represented by

$$
2\left(x^{2}+y^{2}\right)-3 x y+(x+y)+1=z^{2} .
$$

As quadratic equations are rich in variety, one may search for other choices quadratic equation with variables greater than or equal to 3 and determine their properties through special numbers.

\section{REFERENCES}

1. L.E.Dickson, History of Theory of Numbers and Diophantine Analysis, Vol. 2, Dover publications, New York (2005).

2. L.J.Mordell, Diapiophantine equations, Academic press New York (1970).

3. R.D.Carmicheal, The Theory of Numbers and Diophantine Analysis, Dover publications, New York (1959).

4. M.A.Gopalan and D.Geetha, Lattice points on the hyperboloid of two sheets $x^{2}-6 x y+y^{2}+6 x-2 y=z^{2}+4$, Impact J. Sci. Tech., 4 (2010) 23-32.

5. M.A. Gopalan, S.Vidhyalakshmi and A.Kavitha, Integral points on the homogeneous cone $z^{2}=2 x^{2}-7 y^{2}$, The Diophantus J Math., 1(2) (2012) 127-136.

6. M.A.Gopalan, S.Vidhyalakshmi and G.Sumathi, Lattice points on the hyperboloid of one sheet $4 z^{2}=2 x^{2}+3 y^{2}-4$, The Diophantus J Math, 1(2) (2012) 109-115.

7. M.A.Gopalan, S.Vidhyalakshmi and K.Lakshmi, Integral points on the hyperboloid of the two sheets $3 y^{2}=7 x^{2}-z^{2}+21$, The Diophantus J Math., 1(2) (2012) 99-107.

8. M.A.Gopalan, S.Vidhyalakshmi and S.Mallika, Observations on the hyperboloid of one sheet $x^{2}+2 y^{2}-z^{2}=2$, Bessel J Math., 2(3) (2012) 221-226. 
On the Non-Homogeneous Ternary Quadratic Equation $2\left(x^{2}+y^{2}\right)-3 x y+(x+y)+1=z^{2}$

9. M.A.Gopalan, S.Vidhyalakshmi, T.R.Usha Rani and S. Mallika, Integral points on the homogeneous cone $6 z^{2}+3 y^{2}-2 x^{2}=0$, Impact J Sci Tech, 6(1) (2012) 7-13 .

10. M.A.Gopalan, S.Vidhyalakshmi and T.R.Usha Rani, Integral points on the nonhomogeneous cone $2 z^{2}+4 x y+8 x-4 z=0$, Global Journal of Mathematics and Mathematical Sciences, 2(1) (2012) 61-67.

11. M.A.Gopalan, S.Vidhyalakshmi and J.Uma Rani, Integral points on the homogeneous cone, $4 y^{2}+x^{2}=37 z^{2}$, Cayley J Math, 2(2) (2013) 101-107.

12. M.A.Gopalan, S.Vidhyalakshmi and A.Kavitha, Observations on the hyperboloid of two sheets $7 x^{2}-3 y^{2}=z^{2}+z(y-x)+4$, International Journal of Latest Research in Science and Technology, 2(2) (2013) 84-86.

13. M.A.Gopalan and B.Sivagami, Integral points on the homogeneous cone $z^{2}=3 x^{2}+6 y^{2}$, IOSR Journal of Mathematics, 8(4) (2013) 24-29.

14. M.A.Gopalan and V.Geetha, Lattice points on the homogeneous cone $z^{2}=2 x^{2}+8 y^{2}-6 x y$, Indian Journal of Science, 2 (2013) 93-96. 